\title{
Home Self-Training: Visual Feedback for Assisting Physical Activity for Stroke Survivors
}

\author{
Renato Baptista ${ }^{\mathrm{a}, *}$, Enjie Ghorbel ${ }^{\mathrm{a}}$, Abd El Rahman Shabayek ${ }^{\mathrm{a}, \mathrm{b}}$, Florent Moissenet ${ }^{\mathrm{c}}$, Djamila Aouada ${ }^{\mathrm{a}}$, Alice \\ Douchet $^{\mathrm{d}}$, Mathilde André ${ }^{\mathrm{d}}$, Julien Pager ${ }^{\mathrm{d}}$, Stéphane Bouilland ${ }^{\mathrm{d}}$ \\ ${ }^{a}$ Interdisciplinary Centre for Security, Reliability and Trust (SnT), University of Luxembourg, Luxembourg \\ ${ }^{b}$ Computer Science department, Faculty of Computers and Informatics, Suez Canal University, Egypt \\ ${ }^{c}$ Centre National de Rééducation Fonctionnelle et de Réadaptation-Rehazenter, Laboratoire d'Analyse du Mouvement et de la Posture (LAMP), \\ Luxembourg \\ ${ }^{d}$ Fondation Hopale, France
}

\begin{abstract}
Background and Objective: With the increase in the number of stroke survivors, there is an urgent need for designing appropriate home-based rehabilitation tools to reduce health-care costs. The objective is to empower the rehabilitation of post-stroke patients at the comfort of their homes by supporting them while exercising without the physical presence of the therapist.

Methods: A novel low-cost home-based training system is introduced. This system is designed as a composition of two linked applications: one for the therapist and another one for the patient. The therapist prescribes personalized exercises remotely, monitors the home-based training and re-adapts the exercises if required. On the other side, the patient loads the prescribed exercises, trains the prescribed exercise while being guided by color-based visual feedback and gets updates about the exercise performance. To achieve that, our system provides three main functionalities, namely: 1) Feedback proposals guiding a personalized exercise session, 2) Posture monitoring optimizing the effectiveness of the session, 3) Assessment of the quality of the motion.

Results: The proposed system is evaluated on 10 healthy participants without any previous contact with the system. To analyze the impact of the feedback proposals, we carried out two different experimental sessions: without and with feedback proposals. The obtained results give preliminary assessments about the interest of using such feedback.

Conclusions: Obtained results on 10 healthy participants are promising. This encourages to test the system in a realistic clinical context for the rehabilitation of stroke survivors.
\end{abstract}

Keywords: Stroke-survivors, Home-based rehabilitation, Visual feedback, 3D skeleton

\section{Introduction}

The number of stroke survivors in Europe is expected to increase by $25 \%$ in 2035 due to the growth of the aging population as reported in "The Burden of Stroke In Europe" report [1]. Hence, a great effort is being made to

\footnotetext{
${ }^{*}$ Corresponding author

Email address: renato. baptista@uni.lu (Renato Baptista)
}

design solutions aiming at improving the quality of life of stroke survivors.

Thanks to the tremendous scientific and technological progress in computer science (e.g. computer vision, Internet of Things (IoT), machine learning), a wide range of technology has been developed in this direction [2; 3]. More particularly, one can mention systems able to help clinical experts in the long-term rehabilitation process of stroke survivors [4]. However, as reported in [5], there 
exist very few medical institutions exploiting automatic computer-based tools. Thus, the design of a system supporting the rehabilitation of stroke survivors and more generally of disabled individuals is of major importance and can significantly impact medical development.

In a review of human motion tracking systems for rehabilitation, Zhou et al. [6] have highlighted the advantages of using markerless sensors since they present fewer restrictions, achieve good performance and are affordable. In [7], a virtual reality application has been introduced for brain injury rehabilitation. Lin et al. [8] proposed to use an eye-tracking device for rehabilitation of patients with dysfunctional eye movement. Also, in [9; 10], experimental results have proved that virtual reality applications favorably impact rehabilitation, while in [11], the SonyPS2 potential for rehabilitation has been studied. To increase the reliability of such systems, some researchers have chosen to work with a multi-camera system. For instance, Lin et al. [12] employed a double CCD camera to capture the motion during rehabilitation sessions. In [13], the authors made use of a stereo-vision system in order to detect and assess the human motor reactivity under stimulation. On the other hand, some works have reinforced visionbased methods by combining markerless acquisition systems with other kinds of sensors or technologies. For example, Mirelman et al. [14] proposed a robotic-virtual reality integrated system to train post-stroke patients. Similarly, in [15], the authors associated Virtual Reality to Wii gaming technology for stroke rehabilitation.

Recently, the availability of RGB-D cameras (e.g. Kinect) has considerably boosted computer vision-based rehabilitation systems. In addition to RGB images, these cameras are able to capture in real-time depth images and 3D human skeleton sequences [16]. In fact, the 3D human skeleton, considered as a high-level representation, allows better discrimination of motion and is easy to manipulate [16, 17]. Following this trend, researchers used the Kinect for the rehabilitation of individuals with motor disabilities [18; $19,20,21 ; 22 ; 23 ; 24 ; 25]$.

In [19], a game-based rehabilitation application has been developed and the two acquisition systems OptiTrack 11 and Kinect have been compared in this context. Moreover, the authors investigated the capability of the
Kinect as a robust tool for Spinal Cord Injuries (SCI) rehabilitation. The results have shown that the performance of both sensors is comparable. Clark et al. [26] also compared marker-based (VICON2) and markerlessbased (Kinect) systems to assess the lateral trunk lean angle in healthy participants. Using an individualized calibration, the authors were able to obtain a small mean difference of $0.8^{\circ}+/-0.8^{\circ}$. In [20], the authors presented a virtual rehabilitation system for stroke survivors composed of a Kinect and a haptic glove for tactile feedback. Bao et al. [21] introduced a Kinect-based virtual reality training for the upper limbs after subacute stroke. Also, Zannatha et al. [22] proposed a rehabilitation system for stroke survivors by combining Kinect, a humanoid robot and ergonometric signals. Lozano-Quilis et al. [23] have proposed a system using virtual reality and natural user interfaces for the rehabilitation of patients with multiple sclerosis. In [24], a virtual reality-based exergame for post-stroke rehabilitation has been introduced and called Motion Rehab AVE 3D. Recently, Spasojevic et al. [27] presented a Kinect-based application to provide support to medical doctors during the clinical evaluation phase. In [25], a system allowing the therapist to tailor an exercise according to the patient has been proposed.

The aforementioned rehabilitation based systems have been mainly designed to assist clinicians in dedicated centers and are hardly usable remotely (without the physical presence of the therapist) since they do not provide realtime automatic feedback. In fact, to the best of our knowledge, very few works have proposed a computer-based system for home-based rehabilitation.

In this paper, a markerless and affordable home-based rehabilitation system is designed for stroke survivors to orient them during the training is proposed. Figure 1 1 illustrates an overview of the proposed system. Our system is designed as follows:

1. Kinect is chosen for the acquisition as it is a low-cost markerless RGB-D sensor with acceptable and reliable measurement accuracy. In principle, any RGBD sensor can be used.

2. To ensure the continuous communication between the therapist and the patient, our system is composed of two connected applications; one for each.

\footnotetext{
1 https://optitrack.com/
}

$\sqrt[2]{\text { https://www.vicon.com/ }}$ 


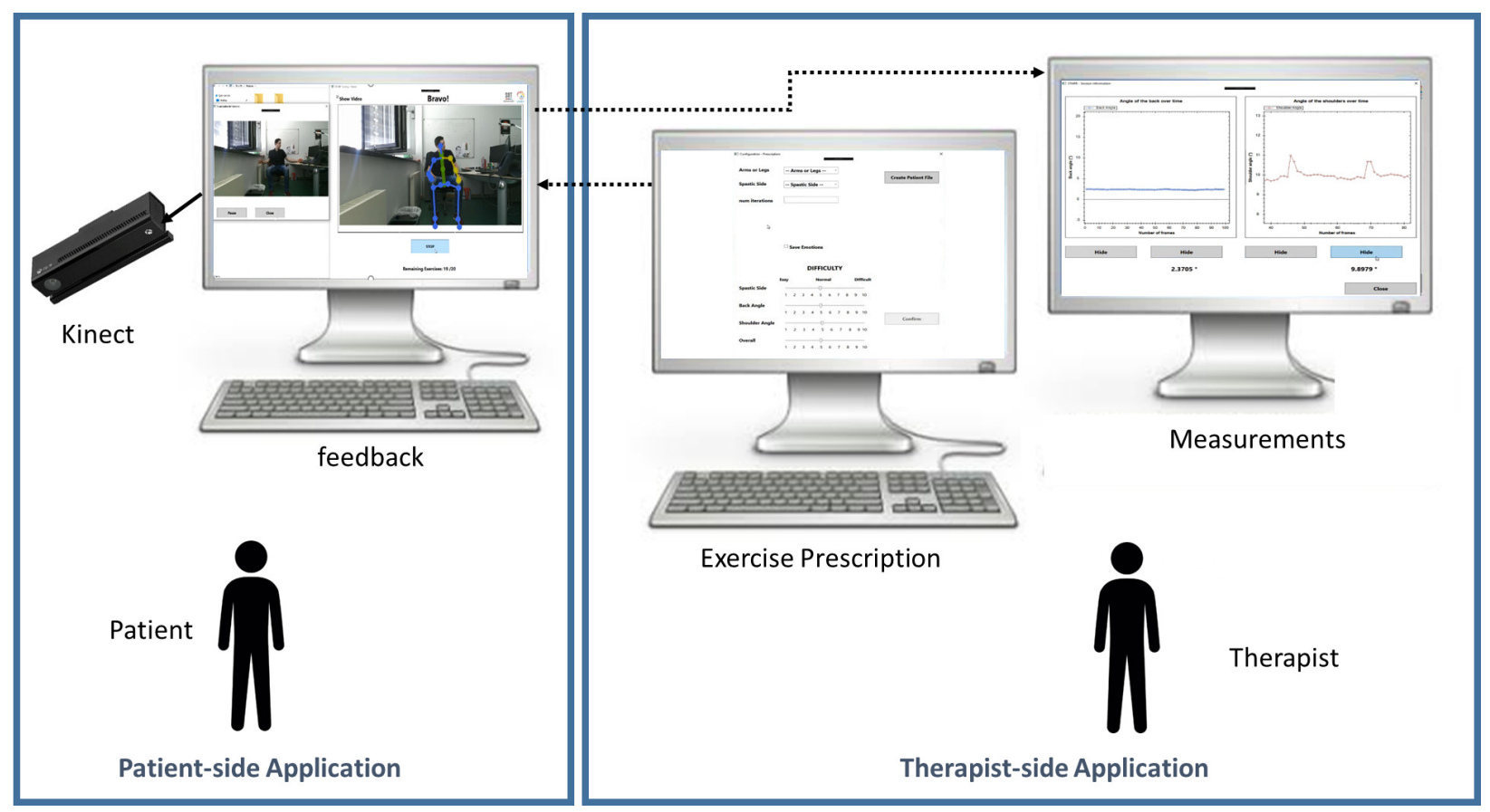

Figure 1: Overview of the proposed system dedicated to stroke survivors. The system consists of the combination of two end-user applications called: 1) the therapist side application; and 2) the patient side application; shown respectively on the left and right sides of the figure. Better visualized in color.

(a) The therapist-side application enables remote personalized prescription of exercises and visualization of the measurements per exercising session.

(b) The patient-side application enables real-time and automatic visual feedback [28, 29, 30, 31; 32] (clinically validated) as well as reporting on how well the exercise is performed [29, 33].

3 . The measurements of each exercising session performed by the patient are automatically communicated to the therapist.

The proposed system exploits our previous work [28 29, 30, 31, 32], where we proposed two different visual feedback techniques to guide stroke survivors in selfrehabilitation scenarios. In summary, we present a system where a number of innovative computer methods were proposed, as listed below:

1. The development of a relevant and instantaneous computer-vision based feedback to guide and sup- port the movement of the patient using a single RGBD sensor;

2. The development of a relevant and instantaneous computer-vision based feedback to monitor the postural defects of the patient using a single RGB-D sensor;

3. The development of a relevant abstract measurement for evaluating the correctness of the global motion of the patient;

4. The optimization of the previously mentioned components in order to have a real-time interaction with the patients;

5. The full home-based training system that is composed of two applications which gathers all the components mentioned above.

As for the clinical point of view, this work has the following contributions:

1. A complete system architecture composed of two applications: the therapist side and the patient side. 
2. A personalized prescription is done by the therapist where (s)he can tailor exercises according to the patient's profile and also update them given the exercising results.

3. A new feedback measurement that quantifies the global quality of the exercise.

4. An evaluation of spastic limb movement by comparing it to the equivalent healthy limb movement, instead of using a given template.

5. A first validation of the proposed system by conducting experiments on 10 healthy participants.

The rest of the paper is structured as follows: Section 2 presents the methodology of the proposed home-based rehabilitation system. Section 3 details the experiments and the results conducted in order to evaluate the system. Section 4 discusses the obtained results. Finally, Section 5 concludes the paper and presents future works.

\section{Methodology}

\subsection{Clinical Motivation}

The primary goal of the proposed system is to support the rehabilitation of stroke survivors at home. Exercising is crucial for them to recover some autonomy in their daily life activities [34]. Unfortunately, many stroke survivors do not exercise regularly due to multiple reasons, such as fatigue, lack of motivation, confidence and skill levels [35].

Traditionally, stroke survivors are initially subject to physical therapy under the supervision of a health professional with the objective of restoring and maintaining activities of daily living known as functional activities in rehabilitation centers [36]. Consequently, they are continuously advised by experts on how to improve their movements and monitored in order to avoid health risks [37]. Unfortunately, due to the high economical burden [37], on-site rehabilitation is generally prescribed for a short period of time and recommended treatments and activities for home-based rehabilitation are suggested [38]. As an alternative, home-based rehabilitation, or selfrehabilitation programs are usually proposed since they are not expensive and do not involve the presence of a therapist [39, 40]. To that aim, the therapist usually explains and demonstrates the exercises to be performed by the patient. In addition, the therapist also provides the patient with a booklet containing an illustrated description of the prescribed exercises [40].

Unfortunately, home-based rehabilitation programs using a booklet present some drawbacks. First, they are generally conducted in parallel with traditional on-site rehabilitation to ensure the effectiveness of the program and consequently require the physical presence of a therapist. Second, the clinician cannot control the home-based exercising because it is not possible to: 1) know if the patient completed the exercises; 2) guide and advise him during the training session; 3 ) detect misconducted exercises that can lead to musculoskeletal injuries, and 4) evaluate the exercise quality. Finally, the exercises illustrated in the booklet [40] are not tailored to patient-specific conditions. As a consequence, there is a need to design appropriate systems for monitoring patients during their exercising at home.

Having this in mind, the proposed system aims to monitor and guide stroke survivors while exercising without the physical presence of the therapist. To ensure adaptive clinical monitoring, continuous communication between the patient and the therapist is maintained allowing the therapist to follow the patient evolution and to adapt the exercises to its specific conditions. Some systems including this communication feature have already been designed as in [25, 41].

However, compared to earlier systems, we propose to include real-time, visual and easily interpretable motion feedback [28, 29, 30] tailored to the therapist prescription. This is different from what exists in the market, as for example, in [25], where feedback in a virtual reality game is proposed in the form of binary feedback, i.e., correct or incorrect action. It is given after each repetition, independently of the therapist prescription. In [42], more sophisticated feedback proposals are introduced. Nevertheless, the corrective feedback is analyzed per joint involving a complex set of instructions for suggesting a particular body-part motion which is hard to interpret by the patient. Thus, the two simple novel color-coded feedback proposals are designed to instantly guide the patient on how to correctly perform the exercise and to warn him to avoid damaging compensatory movements. Compensatory movements are defined as the appearance of new motor patterns resulting from the adaptation of remaining motor elements or substitution [43]. For example, motor 
THERAPIST-SIDE APPLICATION

PATIENT-SIDE APPLICATION

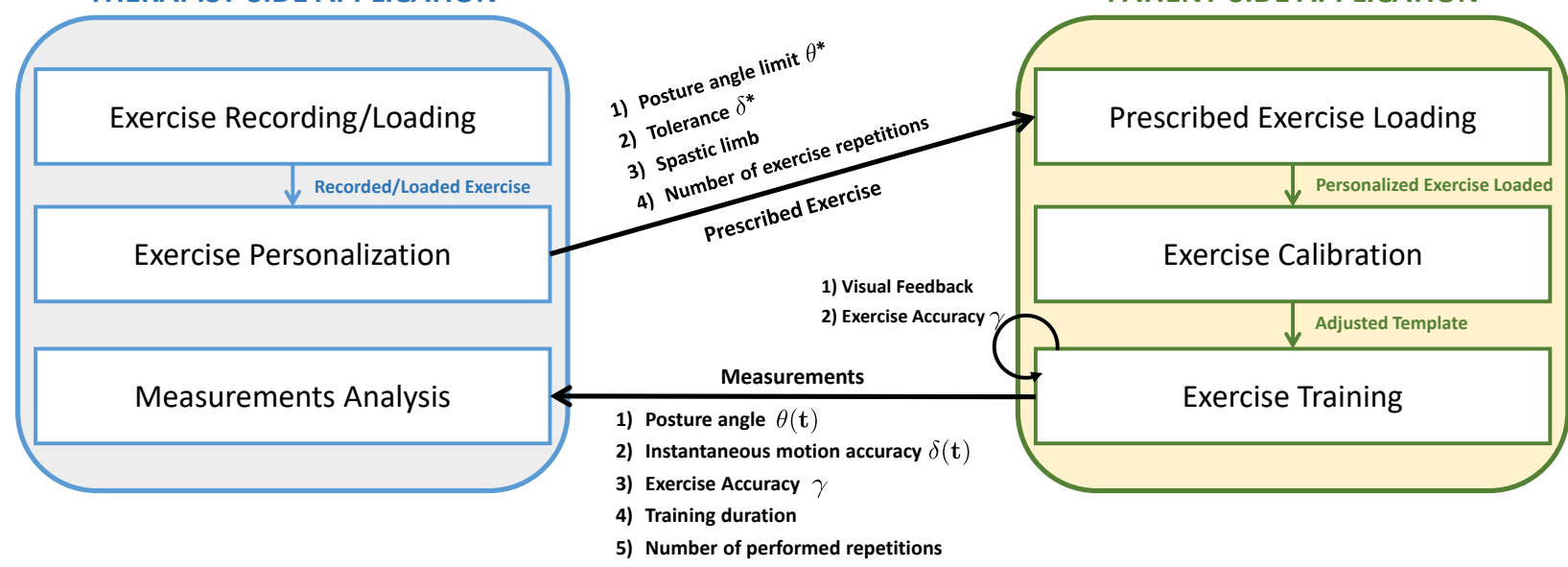

Figure 2: The architecture of the proposed system. Internal communication and functionalities of the proposed system composed of the therapist side application and the patient side application. Better visualized in color.

compensations can relate to the movement patterns that incorporate trunk displacement and rotation, scapular elevation, shoulder abduction, and internal rotation [44, 45].

Moreover, in order to evaluate the global quality of the exercise, we propose a novel abstract measurement to be computed after each repetition. There are different attempts in the literature aiming at quantifying the exercise quality such as [42; 46]. These existent metrics rely on the comparison of the performed exercise with manually predefined templates, which do not take into account the patient's specificities. To overcome this issue, we propose an original way of defining the template: the patient is asked to do the exercise with the equivalent healthy limb; then, the exercise of quality is computed by comparing the spastic limb movement to the equivalent healthy limb movement. Thanks to this process, the anthropometry, as well as the movement particularity of the patient, are taken into consideration allowing a more subtle analysis.

\subsection{System Overview}

The proposed system is designed for home-based rehabilitation of stroke survivors under remote clinical control. For this reason, the proposed system is composed of two linked end-user applications, one for the therapist and one for the patient, as shown in Figure 1 . For more clarity, in the rest of this paper, we will refer to these two applications as the therapist side and the patient side applications.

Using the therapist side application, the therapist transfers a prescription containing personalized exercise(s) to the patient and receives relevant measurement describing each home-based training performed by the patient. For this application, only a basic camera and a computer are needed. The patient side application allows the interpretation of the therapist prescription, the presentation of visual feedback to the patient while training and transferring the training measurement data to the therapist. Figure 2 depicts in more detail the global architecture of the system.

For a deeper understanding of the system, we propose to describe a typical usage scenario of the system constituted of 6 steps: 1) the therapist starts by recording a video regarding a specific rehabilitation exercise; 2) the prescription or in other words, the exercise personalization, is created based on the patients profile. Then, the prescription is automatically sent to the patient; 3) starting from the moment that the patient receives the prescription, the patient loads the exercise personalization that was prescribed by the therapist; 4) the patient is first asked to perform a calibration task. Such a calibration task corresponds to the exact same exercise that was prescribed but with the healthy limb. This calibration process allows to adapt the exercise constraints and parameters to the anthropometry of the patient; 5) the patient starts training 
his spastic limb. To improve and correct his movement, the patient is constantly oriented with visual and understandable feedback proposals while training; and 6) at the end of the training session, a report containing relevant measurements describing the quality of the spastic limb motion is communicated to the therapist.

In Section 2.3 and Section 2.4, the architecture of the therapist and the patient side applications are detailed.

\subsection{Therapist Side Application}

As illustrated in Figure 2, the therapist side application is formed by three main components: 1) record/load an exercise; 2) prescribe a personalized exercise, and 3) analyze measurements.

\subsubsection{Exercise Recording/Loading}

The therapist loads or records rehabilitation exercises that can be prescribed to a particular patient. The recorded/loaded exercise constitutes a reference video which shows an exercise performed by a therapist.

\subsubsection{Exercise Personalization}

After choosing the exercise to be prescribed, the therapist adapts it according to patient-specific conditions by adjusting the following parameters:

- Maximum Posture angle $\left(\theta^{*}\right)$. It represents the maximum back angle with respect to the vertical plane for which the posture of the patient is considered as acceptable;

- Spastic limb. It specifies the spastic body-part to be trained;

- The number of exercise repetitions prescribed to the patient $\left(n_{1}\right)$;

- Tolerance $\left(\delta^{*}\right)$. It defines the overall tolerated error. The lower the tolerance is, the stricter the feedback proposals are.

\subsubsection{Measurements Analysis}

This functionality allows the therapist to follow and analyze the home-based training sessions without requiring the physical presence of the patient. Each time that the patient performs a training session, a detailed report is sent to the therapist through dedicated communication service, as shown in Figure 2. This report contains the following measurements which are detailed in Section 2.4

- Posture angle $\theta$. It represents the back angle with respect to the vertical plane during the whole training session;

- Instantaneous exercise accuracy $\delta(t)$ at each instant $t$ of the spastic limb;

- Duration of the training session;

- Number of repetitions $\left(n_{2}\right)$ of the exercise performed by the patient during the training session;

- Exercise accuracy $\gamma$. It quantifies the quality of the patient motion based on the temporal alignment proposed in [33].

Such measurements allow the therapist to assess and evaluate the progress of the patient while using the proposed home-based rehabilitation system. This would also allow the therapist to have a clear understanding of the patient progress and to adapt the parameters to better fit the rehabilitation of the patient.

\subsection{Patient Side Application}

The patient side application enables the patient to load the prescribed exercise, calibrates the exercise with respect to the healthy limb, and train the spastic limb, as depicted in Figure 2 .

\subsubsection{Prescribed Exercise Loading}

The patient should load the prescribed exercise that was created and shared by the therapist. This allows the system to set up all the movement constraints that are related to the patient. Such constraints directly define how strict should be the feedback proposals to be provided and also the number of repetitions asked by the therapist.

\subsubsection{Exercise Calibration}

This step is necessary to create the reference motion allowing the evaluation of the movement the spastic limb. The patient is therefore asked to calibrate the system with the equivalent healthy limb. For example, let us assume that a given patient has a spastic right arm and that the therapist has consequently prescribed some exercises involving it. Then, during the calibration phase, the patient 


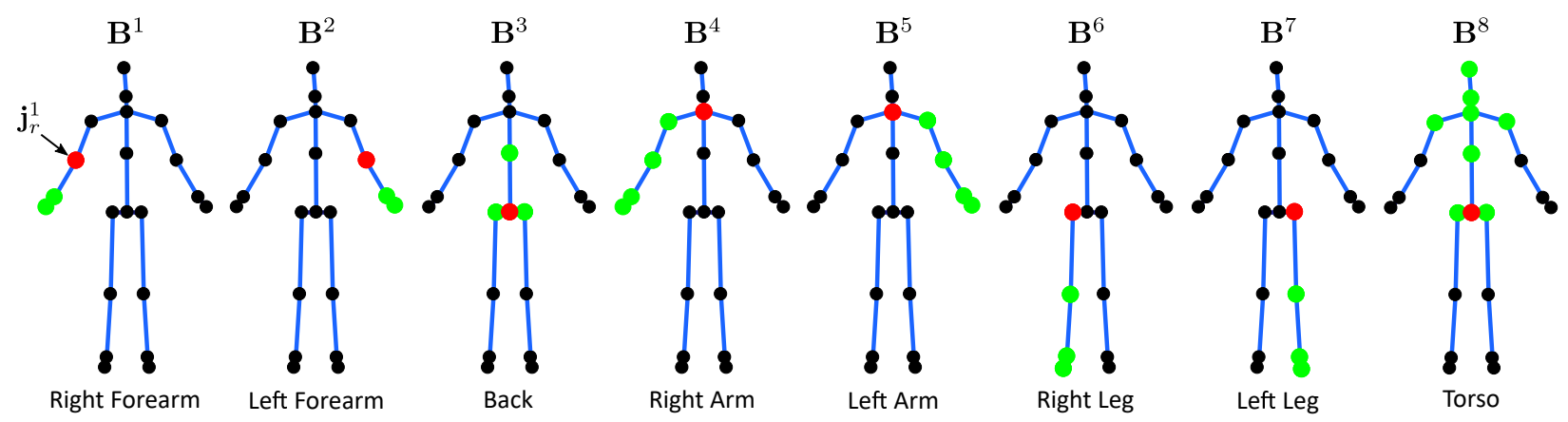

Figure 3: Body-part representation. The set of joints for each body-part is highlighted in green and its corresponding local coordinate system in red color. The notation for each body-part is shown above the respective skeleton. Better visualized in color.

is asked by the system to do exactly the same exercise with the left arm.

Since we make use of an RGB-D sensor, the 3D skeleton information extracted from depth images [16] is used to capture the movement during the calibration and later during the training session. Not very accurate skeletons encode the human pose (in contrast to RGB or depth images). Thus, the human body motion is represented using the spatial position of the skeleton joints, e.g. left and right shoulders, elbows, wrists, etc. At each instant $t$, let $S(t)=\left[j_{1}(t), \cdots, j_{N}(t)\right]$ denote the captured skeleton composed of $N$ joints, where each $j_{i}(t) \in \mathbb{R}^{3}$ represents the estimated 3D position of the joint $i$. For optimal use of the skeleton, it is important to overcome skeleton viewpoint variation, camera positioning variability, and anthropometry variation. Consequently, the same preprocessing of skeleton normalization and spatial alignment presented in [28, 29; 47] is applied to the skeleton sequence.

To differentiate it from the rest of the skeleton, we denote the body-part of interest by $B^{\text {limb }}(t)=$ $\left[j_{1}^{l i m b}(t), \cdots, j_{n_{\text {limb }}}^{\text {limb }}(t)\right]$, which is composed of $n_{\text {limb }}$ joints. Figure 3 shows the body-part representation that is used throughout the paper.

Hence, in the calibration step, the joint positions of the side-opposed limb are recovered. To make them comparable to the spastic limb, we apply an axial symmetry. The obtained trajectories, varying over time $t$, representing the reference motion are denoted by $\hat{B}_{\text {limb }}(t)$.

\subsubsection{Exercise Training}

While training, the patient is guided in how to correctly perform the proposed exercise and to avoid movement compensation. Consequently, the feedback proposals can be divided into three distinct parts: 1) the instantaneous motion feedback; 2) the posture monitoring, and 3) the measure of the exercise accuracy. While the two first feedback proposals are visually provided to the patient in realtime, the last one is reported to the patient as a percentage score after finishing each training iteration.

\section{Instantaneous Motion Feedback}

The objective of motion feedback is to support the patient while performing the prescribed exercise. To that end, the feedback proposals are provided at each time instant in order to iteratively help the patient improving the movement of the body-part of interest.

First, to measure the similarity between the reference body-part movement $\hat{B}^{\text {limb }}(t)$ and the spastic body-part movement $B^{\operatorname{limb}}(t)$ at each instant $t$, the Euclidean distance between them is used as the score function $m(t)$ as depicted in (1).

$$
m(t)=\sum_{i=1}^{n_{\text {limb }}}\left\|j_{i}^{l i m b}(t)-\hat{j}_{i}^{\text {limb }}(t)\right\|^{2} .
$$

Hence, the joints of the spastic and the reference bodyparts respectively denoted as $j_{r}^{\text {limb }}$ and $\hat{j}_{r}^{\text {limb }}$ (colored in red in Figure 3 are anchored in the same local coordinate system. Consequently, the aim is to compute the ro- 
tation $\mathbf{R} \in \mathrm{SO}(3)$ that minimizes the following error:

$$
e_{\mathbf{R}}(t)=\sum_{i=1}^{n_{\text {limb }}}\left\|\mathbf{R} j_{i}^{l i m b}(t)-\hat{j}_{i}^{l i m b}(t)\right\|^{2} .
$$

Thus, at each instant $t$, the intensity cost $\delta(t)$ defined as

$$
\delta(t)=m(t)-e_{\mathbf{R}}(t)
$$

relates to the difference between the score function $m(t)$ and the error $e_{\mathbf{R}}(t)$.

For a given body-part $k$, Figure 4 shows an example of the intensity cost $\delta(t)^{k}$ for the movements of clapping and waving hands, where the cost is computed for each instant $t$. For the alignment of both skeleton sequences, Dynamic Time Warping (DTW) as reported in our previous work [28].

Compared to [28], only the body-part of interest is taken into account. Thus, the feedback is presented by highlighting the body-part of interest (in this case, the spastic limb) using $\operatorname{Color}_{\mathbf{B}^{\text {limb }}}(t)$, which is computed based on the intensity cost $\delta(t)$ as in [30]. It is defined as

$$
\operatorname{Color}_{B^{\text {limb }}}(t)=\left\{\begin{array}{ll}
\text { green } & , \text { if } \delta(t) \leq \delta^{*}, \\
\text { red } & , \text { otherwise }
\end{array},\right.
$$

where the threshold $\delta^{*}$ represents the tolerance fixed by the therapist during the exercise personalization phase. Note that, $\delta^{*}$ plays an important role during the exercise personalization. This parameter is highly correlated with the feedback that is provided to the patient while exercising. Considering that the feedback proposals are presented based on the reference motion (refer to Section 2.4.2, there is a need to sample the reference motion with the objective of guiding the patient to iteratively perform the proposed motion. As for the sampling function, we assumed a uniform distribution of the reference motion. Consequently, the parameter $\delta^{*}$ defines the threshold that the patient needs to reach in order to sequentially advance on the uniform distribution of the reference motion. In fact, if $\delta^{*}$ is relatively high, the provided feedback might be irrelevant. This motivates the interaction between the therapist and the patient. The more the therapist knows the limitations of the patient (by fixing the threshold $\delta^{*}$ accordingly), the more suitable the system is. The color transition from green to red (or vice-versa) is done gradually based on the current value of $\delta(t)$, as shown in Figure 6(c) (from [30]). The green color feedback expresses the correctness, while the red one indicates the inverse.

\section{Posture Feedback}

The posture monitoring feedback has been designed in order to avoid postural compensation in real-time [29]. As mentioned in Section 2.1, when stroke survivors are asked to perform specific movements, they tend to use the trunk to help in performing the movement. It is undesired since it can induce musculoskeletal injuries. Thus, the posture feedback is given using a feature reported in our earlier work [29], which checks if the patient keeps a straight back aligned with the gravity vector. Figure 5(a) illustrates the patient's body representation divided by the plane of symmetry. The spine vector $w$ is defined as the vector that connects the hip joint, which is also the origin of the world coordinate system, to the neck joint, as illustrated in Figure 5(b) Considering that the skeletons are previously normalized and aligned so that the $z$-axis is aligned with the gravity vector, analyzing the deviation from a correct back posture is achieved by computing the angle $\theta$ between $\mathbf{w}$ and the vertical direction $\mathbf{z}$ corresponding to the $z$-axis:

$$
\theta=\angle(\mathbf{w}, \mathbf{z})
$$

Thus, the higher the angle $\theta$ is, the worse the back posture is. Taking into consideration the clinical practices, the error of the angle that is introduced by the Kinect is of high importance. In [26] the authors evaluated the lateral trunk lean angle during the gait training using two different setups: 1) marker-based system (VICON); and 2) markerbased system (Kinect). In this study, the mean error of the Kinect sensor was of $3.2^{\circ}+/-2.2^{\circ}$ when compared to the VICON system. However, with an individualized calibration, the authors were able to reduce the mean error to $0.8^{\circ}+/-0.8^{\circ}$. According to [48], such a range of error is acceptable for clinical gait analysis. Furthermore, they suggest that errors that are not larger than $5^{\circ}$ may not have an impact on clinical interpretation. While these results can not be directly applied to the postural angle measured in the present study, it provides the first threshold of clinical acceptability. 


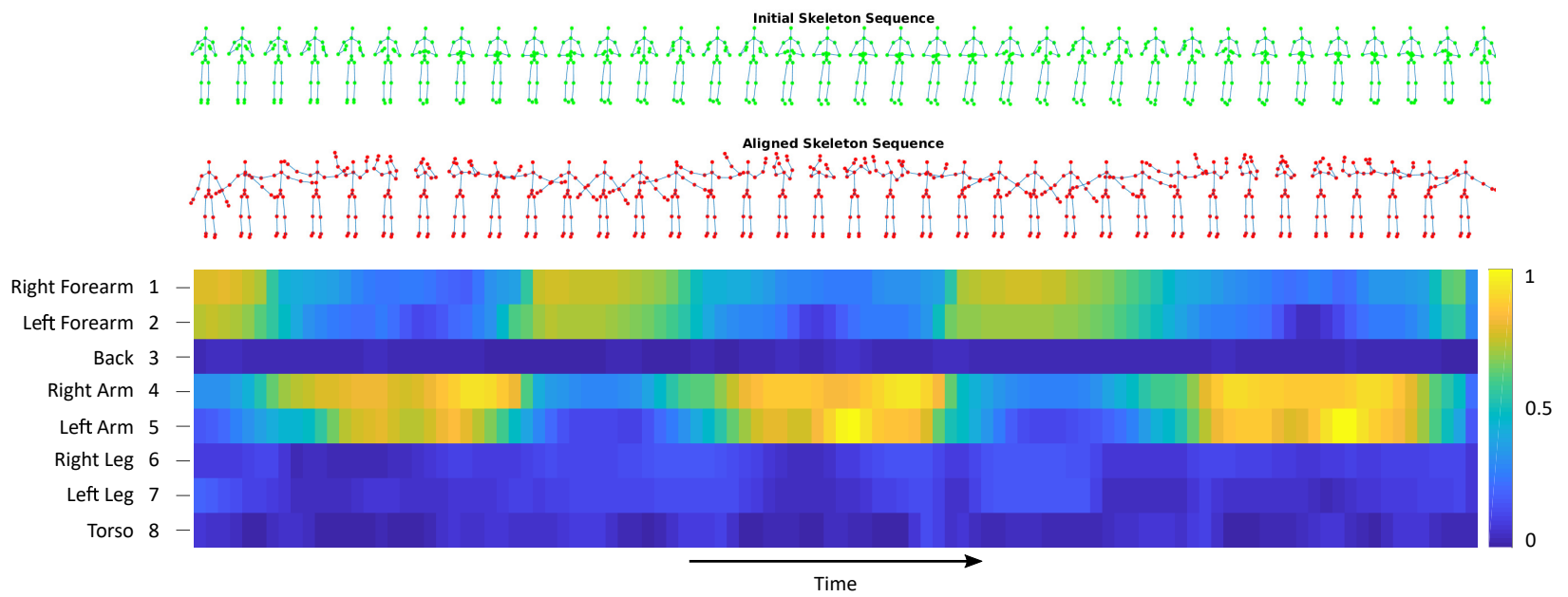

Figure 4: Intensity cost $\delta(t)^{k}$ of the feedback required for each body-part $k$. (Top) Skeleton sequence performing the clapping movement; (middle) skeleton sequence corresponding to the waving movement using two hands after spatial and temporal alignment; and (bottom) the intensity cost $\delta(t)^{k}$ calculated for each temporal instant independently (the vertical axis corresponds to different body-parts, while the horizontal axis regards the temporal dimension). Better visualized in color.

Considering the feature $\theta$, the posture monitoring feedback is also presented in a real-time color-based way. In this case, the highlighted body-part is always the back denoted by $B^{\text {back }}(t)$ and $\mathbf{B}^{3}$ in Figure 3 The color-based feedback Color $_{B^{b a c k}}(t)$ at each instant $t$ is defined as

$$
\operatorname{Color}_{B^{b a c k}}(t)=\left\{\begin{array}{ll}
\text { green } & , \text { if } \theta \leq \theta^{*} \\
\text { red } & \text {, otherwise }
\end{array},\right.
$$

where $\theta^{*}$ is the threshold fixed by the therapist during the exercise personalization step. This threshold indicates the maximum allowable angle to consider the patient posture as correct. The transition from one color to other changes gradually with the value of the current angle $\theta$ as presented in Figure 6(c)

\section{Exercise Accuracy}

In contrast to the two previous types of feedback, this one is reported to the patient after performing the prescribed exercise. While the previous motion feedback and posture feedback instantly give an indication about the correctness of the local movement, the exercise accuracy provides a global evaluation. Since the skeleton joints of each body-part are provided, the movement of each body-part can be seen as a set of joints spatially varying over time. Thus, they can be considered geometrically as a set of joint trajectories. Thus, to evaluate the quality of the spastic body-part movement $B_{\text {limb }}^{\text {norm }}$, we propose to compare it to the movement of the healthy equivalent body-part $\hat{B}_{\text {limb }}^{\text {norm }}$ assimilated to joint trajectories as well. This is done by computing the similarity between each pair of equivalent spastic and healthy joint trajectories. This is an original approach as compared to the common one where a template is used as a reference. This further contributes to the system capability for personalization as the proposed accuracy is relative to each patient and not to an absolute measurement. Indeed, the patient specificities such as anthropometry or the way that the patient performs movements are considered.

Despite this, the execution rate variability resulting from different ways of performing a given movement can bias this comparison. For this reason, we propose to employ Time Variable Replacement (TVR) method of [33], which reduces this rate variability impact. This method reparametrizes the numerical joint trajectories by changing the time variable by a rate-invariant variable. We illustrate the concept of the temporal normalization TVR in Figure 7 Indeed, before applying the normalization, the movement of the reference body-part (blue) and the 


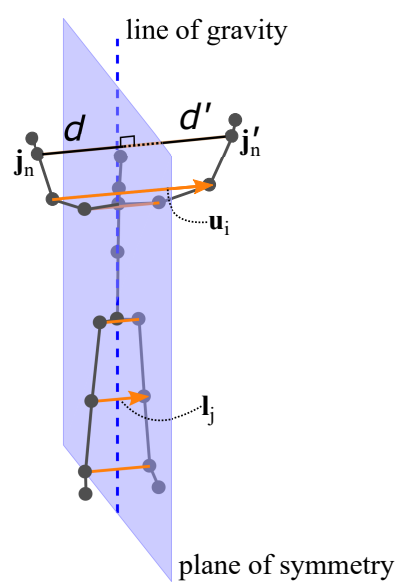

(a)

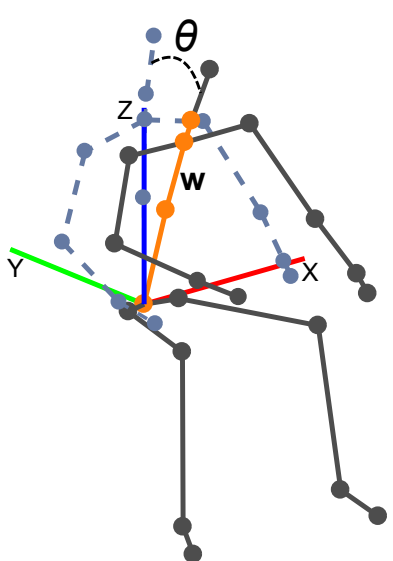

(b)
Figure 5: Human body representation with respect to the plane of symmetry (Figure 5(a) and with respect to the line of gravity (Figure 5(b) angle $\theta$ between the spine vector $\mathbf{w}$ (orange color) and the gravity vector (blue color represents the $z$-axis). Better visualized in color.

movement of the spastic one (green) are expressed in different temporal ranges, making them difficult to compare (Figure 7(a)p. After normalization, the two movements are reported to the same range with a similar distribution of movement over time (Figure 7(a)p. In this work, we used the Normalized Pose Motion Signal Energy (NPMSE) proposed by [33]. The obtained normalized trajectories corresponding to the joint $j$ of the reference and the spastic body-parts are respectively denoted as $\hat{j}_{i}^{\text {limb,norm }}$ and $j_{i}^{\text {limb,norm }}$. To compute the similarity between the two body-parts, the Euclidean distance $D$ between each couple of equivalent joints belonging to the spastic and the reference body-parts is then computed as

$$
D=\sum_{i=1}^{n_{\text {limb }}}\left\|\hat{j}_{i}^{\text {limb }, \text { norm }}-j_{i}^{\text {limb,norm }}\right\|_{2} .
$$

Thus, the smaller the distance is, the more similar the reference and spastic movements are and the better the movement quality of the spastic body-part is.

For an easier interpretation of this distance by the patient, the exercise accuracy measurement is defined as a

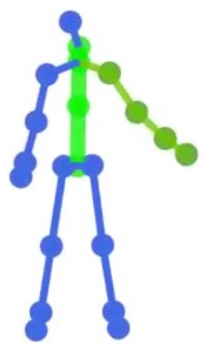

(a)

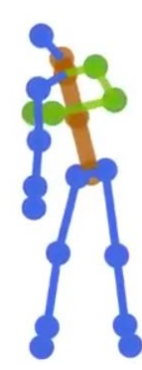

(b)

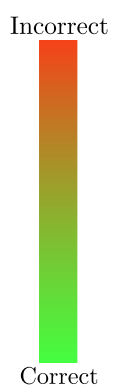

(c)
Figure 6: Feedback proposals in terms of the color code. Figure 6(a) illustrates the correct position of the body-parts of interest and a good posture. On the other hand, Figure 6(b) shows an example in which the patient uses the back to compensate the movement, resulting in red color feedback. Figure 6(c) depicts the color range used in the application to model correct and incorrect body position and bad posture. Better visualized in color.

percentage $\gamma$ computed as

$$
\gamma=\left\{\begin{array}{ll}
100 \times \frac{\delta^{*}}{D} & \text { if } \delta^{*} \leq D \\
100 & \text { otherwise }
\end{array} .\right.
$$

We recall that $\delta^{*}$ represents the tolerated error adjusted by the therapist while prescribing the exercise.

\section{Results}

In this section, the preliminary results realized on 10 healthy subjects in order to evaluate the color-based feedback are reported. In the following, the implementation details, the experimental protocol, as well as the results, are detailed.

\subsection{Implementation Details}

The proposed system has been implemented in Visual C\# within the Windows Presentation Foundation (WPF) framework where XAML, an XML-based language, was used to define and link the interface elements. To run any of the applications, a standard PC with Windows 10 as an operating system is required.

Description of setup: Microsoft Kinect (version 2) sensor connected to the laptop. The system runs at an average frame rate of 25 frames per second (fps). 


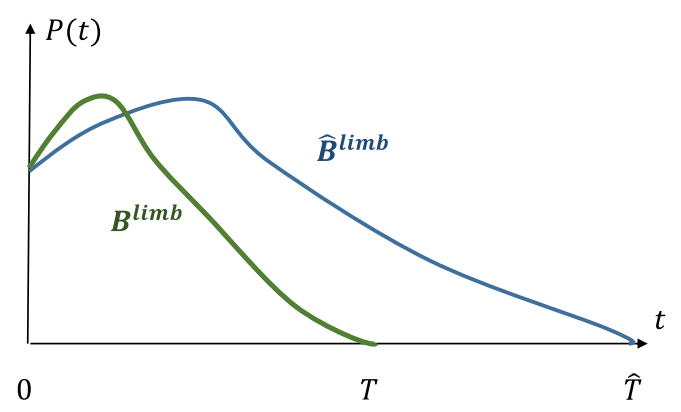

(a)

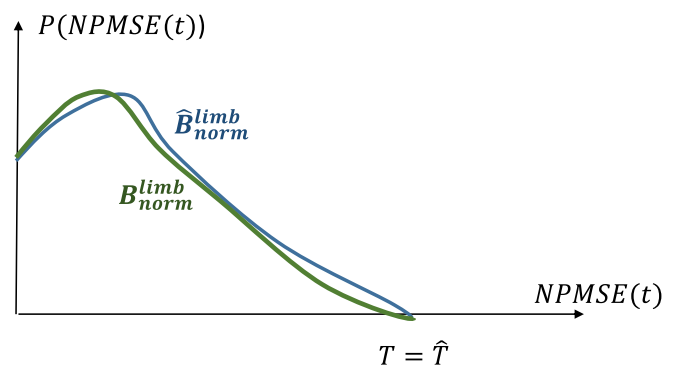

(b)

Figure 7: Example of the TVR method effect on two similar trajectories with different execution rate. While the green trajectory represents the motion of the spastic body-part, the blue one symbolizes the motion of the reference body-part. Figure 7(a) the trajectories are plotted as functions of time. We can observe that the support of the two functions is different $(\hat{T} \neq T)$. Figure $7(\mathrm{~b})$ after the change of the variable time by NPMSE, it can be noted that the trajectories vary in the same range $[0, T]$ and the two trajectories encoding similar movements look more similar. Better visualized in color.

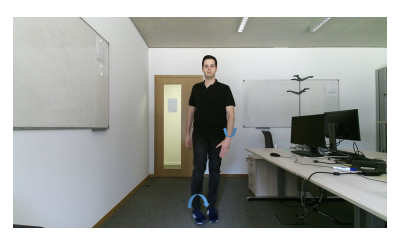

(a)

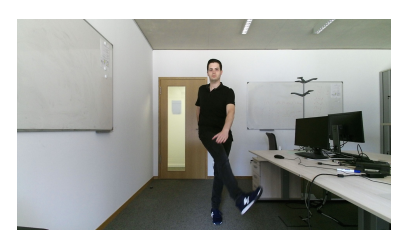

(b)

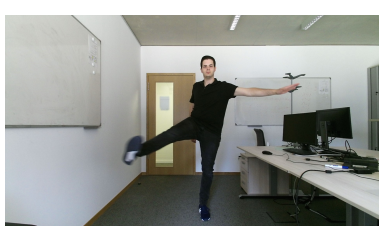

(c)

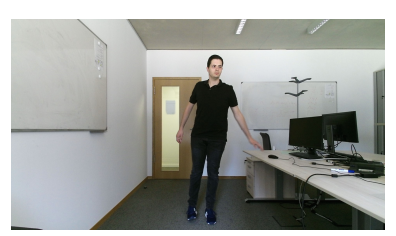

(d)

Figure 8: Illustration of the exercise used for the experiments: (a)-(d) are organized in the chronological order. Better visualized in color.

\subsection{Experimental Protocol}

The system is evaluated on 10 healthy participants. To analyze the impact of the color-based proposed feedback, we carry out two different experimental sessions (without and with feedback). During both sessions, the participants are asked to perform a unique exercise described in Figure 8. Advised by clinicians, the predefined exercise has been chosen to be relatively difficult compared to the classical exercises usually prescribed to post-stroke patients [40]. Indeed, since the system has been tested only on healthy participants, the exercise should ensure a sufficient level of complexity in order to make it somehow challenging. Thus, we propose an exercise that consists of rotating simultaneously the right hand and the left leg from the interior to the exterior with respect to the human body. Indeed, it requires limb coordination due to the fact that we are proposing to move opposite limbs in a circular movement.

First session: Before the beginning of the session, we ex- plain and demonstrate the proposed exercise to each participant. Then, after assimilating the exercise, the participant is asked to repeat the same exact exercise five times. During the session, all information about the 3D skeleton joint positions are acquired using the Kinect sensor and stored in a file. However, no feedback is provided to the participant in this first session.

Second session: The second training session consists of guiding the participant while performing the exercise. For that end, color-based feedback is presented to each participant. Following the same protocol described in the first session, each participant performs the same exercise five times, but now feedback is presented.

\subsection{Experimental Results}

For each participant, we average the exercise accuracy $D$ and the postural angle $\theta$. Figure 9 and Figure 10 respectively report these two values. In Figure 9, we specify that we report the distance $D$ and not the percentage. Thus, 


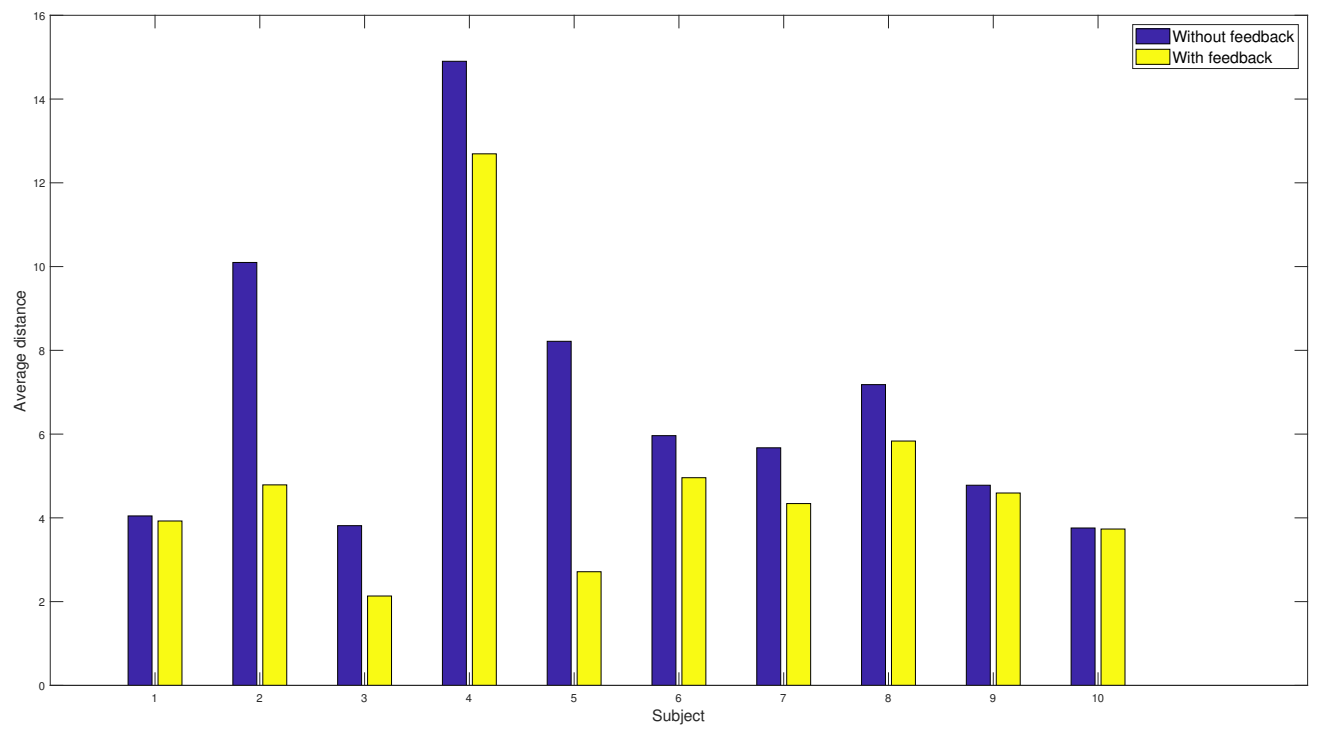

Figure 9: Average of the exercise accuracy for each subject. We specify that the reported value is computed as a distance and not as a percentage using Eq. (7). Better visualized in color.

the higher is the distance, the worse is the exercise accuracy. Therefore, it can be noted that exercise accuracy is improved when the color-based feedback is presented to the participant. Similarly, the postural angle $\theta$ decreases when the color-based feedback is provided (for almost all subjects, except for subject 10).

\section{Discussion}

The aim of this work is to develop a novel home-based system dedicated to stroke survivors using a Kinect sensor. Compared to earlier systems [25, 27, 35, 42, 49], the main clinical originality of our system resides in: 1) the provided color-based feedback, and 2) the continuous communication between the patient and the therapist, allowing an interactive personalization of the exercise, and 3) the quantification in terms of the exercise accuracy. While in computer methods point of view, our system presents innovations in the development and optimization of computer-vision based feedback to guide and monitor the patients' posture while exercising using a single RGB$\mathrm{D}$ sensor. Still in the same aspect, this work also presents an abstract measurement to evaluate the correctness of the patients' movement and the full home-based training system that is composed of two dedicated applications.

In [42], feedback is provided by specifying the joint that (s)he should move as well as the recommended movement direction. This results in a complex set of instructions hardly understandable in real-time by the user. Similarly, Ferda et al. [35] proposed assistive feedback by manually specifying constraints on a single joint which define the correctness of the motion. In contrast, our system does not provide feedback related to a single joint but to a full body-part. This allows the definition of more simple feedback proposals which are directly translated into a simple color affecting the desired body-part (spastic limb). This intuitive message is therefore easy to interpret by the patients. Furthermore, no constraints are imposed while the patients are exercising.

Considering the second aspect, in the works [25, 49] the configuration that is done by the therapist happens when both therapist and patient are facing the game. Consequently, the therapist adapts the movement constraints 


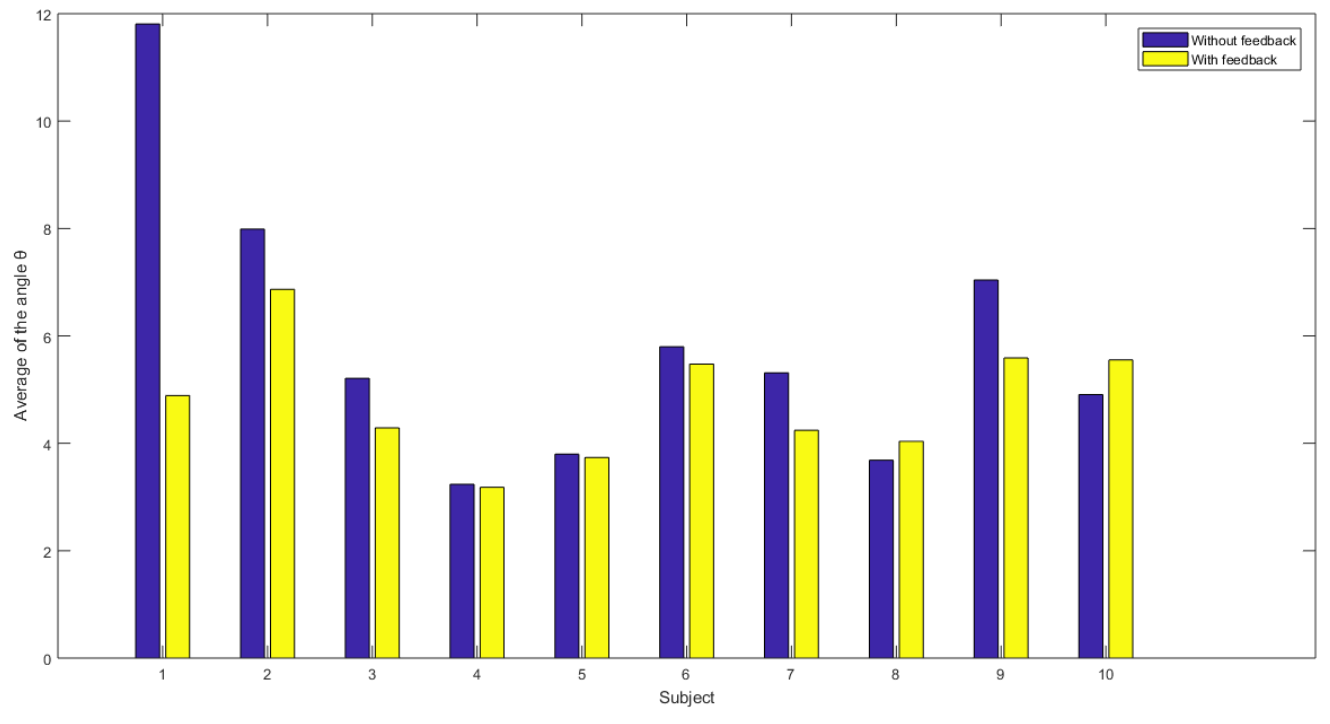

Figure 10: Average of the postural angle $\theta$ (in degrees) for each subject. Better visualized in color.

based on the limitations of the patient with respect to the game environment, while in [27], the authors presented a tool to support the evaluation of the patients. In contrast to these works, our continuous communication happens without the need for the physical presence of the therapist. This allows the therapist to configure remotely the parameters related to the patient's current condition.

The last challenge addressed in this paper is the assessment of the quality of the movement. Compared to previous approaches [46, 50, 51], the proposed system has the advantage of being fully automated and adaptive to the patient's conditions. For instance, in [46], the authors presented a method that quantitatively evaluates muskuloskeletal disorders of patients who suffer from Parkinson's disease. Their system may not be generalized for all kinds of exercises, this is due to their feature selection that is movement dependent (stepping time, swing level of the hands, etc). Tao et al. [50] also proposed a quality assessment framework. They model the dynamics of the human motion by using Hidden Markov Models (HMM). In addition, a manifold-based dimensionality reduction is applied to the human motion sequence. Contrary to that, we do not apply any dimensionality reduction method. In- stead, based on the exercise personalization, we restrict the human body to the specific body-part and we only assess the quality with respect to that body-part. Not only that, but we also achieve real-time measurements.

In addition to the depicted novelties proposed by our system, to the best of our knowledge, we are the first proposing an experimental validation of this feedback. The obtained results on 10 healthy show an improvement of the posture and of the quality of the motion. Nevertheless, with such modest results, we were not able to draw final conclusions. More specifically, the improvement of the posture remains very slight, since the participants are healthy, do not present spastic limbs and consequently do not try to compensate with their back. Notwithstanding, these results allow us to present preliminary assessments about the interest of using such feedback proposals. As for the exercise accuracy, we noted a decrease in the measured distance (refer to Section 2.4.3 - Exercise Accuracy) for all the participants while using the home-based rehabilitation system. These first results tend to demonstrate the interest of the approach but should be applied to further participants, and more particularly to stroke survivors. 


\section{Conclusion}

In this paper, a novel home-based training system for the rehabilitation of stroke survivors has been introduced. Advised by clinicians, our system has been designed to answer different medical requirements. More specifically, its originality consists of: 1) a permanent remote communication between the therapist and the patient (exercise prescription and patient monitoring); 2) novel easilyunderstandable color-based feedback proposals to guide the patient during the training sessions; and 3) the consideration of the patient's anthropometry and specificities to evaluate the quality of the spastic body-part movement (during the personalized prescription and thanks to the calibration phase). Experiments realized on 10 healthy participants shown promising results in order to further test the system for the rehabilitation of stroke survivors. The system is currently tested and evaluated in a clinical pilot study. The results will be reported in a dedicated article.

\section{Acknowledgements}

This work has been funded by the European Union's Horizon 2020 research and innovation project STARR under grant agreement No.689947. Many thanks to our project partners and to the participants who contributed to the system evaluation.

\section{Conflicts of Interest}

The authors of this paper declare no conflicts of interest.

\section{References}

[1] f. t. S. A. f. E. King's College London, "Report the burden of stroke in europe," 2017.

[2] R. Li, B. Lu, and K. D. McDonald-Maier, "Cognitive assisted living ambient system: a survey," Digital Communications and Networks, vol. 1, no. 4, pp. 229-252, 2015.
[3] M. C. Domingo, "An overview of the internet of things for people with disabilities," Journal of Network and Computer Applications, vol. 35, no. 2, pp. 584-596, 2012.

[4] M. A. Musen, B. Middleton, and R. A. Greenes, "Clinical decision-support systems," in Biomedical informatics, pp. 643-674, Springer, 2014.

[5] D. González-Ortega, F. Díaz-Pernas, M. MartínezZarzuela, and M. Antón-Rodríguez, "A kinectbased system for cognitive rehabilitation exercises monitoring," Computer methods and programs in biomedicine, vol. 113, no. 2, pp. 620-631, 2014.

[6] H. Zhou and H. Hu, "Human motion tracking for rehabilitationa survey," Biomedical Signal Processing and Control, vol. 3, no. 1, pp. 1-18, 2008.

[7] P. Wang, I. A. Kreutzer, R. Bjärnemo, and R. C. Davies, "A web-based cost-effective training tool with possible application to brain injury rehabilitation," Computer methods and programs in biomedicine, vol. 74, no. 3, pp. 235-243, 2004.

[8] C.-S. Lin, C.-C. Huan, C.-N. Chan, M.-S. Yeh, and C.-C. Chiu, "Design of a computer game using an eye-tracking device for eye's activity rehabilitation," Optics and lasers in engineering, vol. 42, no. 1, pp. 91-108, 2004.

[9] R. M. E. M. da Costa and L. A. V. de Carvalho, "The acceptance of virtual reality devices for cognitive rehabilitation: a report of positive results with schizophrenia," Computer Methods and Programs in Biomedicine, vol. 73, no. 3, pp. 173-182, 2004.

[10] J. A. Edmans, J. R. Gladman, M. F. Walker, et al., "Validity of a virtual environment for stroke rehabilitation," Stroke, 2006.

[11] D. Rand, R. Kizony, and P. L. Weiss, "Virtual reality rehabilitation for all: Vivid gx versus sony playstation ii eyetoy," in 5th Intl. Conf. On Disability, Virtual Environments and Assoc. Technologies, pp. 8794, 2004.

[12] C.-S. Lin, T.-C. Wei, A.-T. Lu, S.-S. Hung, W.-L. Chen, and C.-C. Chang, "A rehabilitation training 
system with double-ccd camera and automatic spatial positioning technique," Optics and Lasers in Engineering, vol. 49, no. 3, pp. 457-464, 2011.

[13] M. I. Vousdoukas, P. Perakakis, S. Idrissi, and J. Vila, "Svmt: A matlab toolbox for stereo-vision motion tracking of motor reactivity," Computer methods and programs in biomedicine, vol. 108, no. 1, pp. 318-329, 2012.

[14] A. Mirelman, B. L. Patritti, P. Bonato, and J. E. Deutsch, "Effects of robot-virtual reality compared with robot alone training on gait kinetics of individuals post stroke," in 2007 Virtual Rehabilitation, pp. 65-69, Sept 2007.

[15] G. Saposnik, R. Teasell, M. Mamdani, J. Hall, W. Mcilroy, D. Cheung, K. E. Thorpe, L. G. Cohen, and M. Bayley, "Effectiveness of virtual reality using wii gaming technology in stroke rehabilitation: A pilot randomized clinical trial and proof of principle," Stroke, vol. 41, no. 7, pp. 1477-1484, 2010.

[16] J. Shotton, T. Sharp, A. Kipman, A. Fitzgibbon, M. Finocchio, A. Blake, M. Cook, and R. Moore, "Real-time human pose recognition in parts from single depth images," Communications of the ACM, vol. 56, no. 1, pp. 116-124, 2013.

[17] M. Antunes, D. Aouada, and B. Ottersten, "A revisit to human action recognition from depth sequences: Guided svm-sampling for joint selection," in 2016 IEEE Winter Conference on Applications of Computer Vision (WACV), pp. 1-8, IEEE, 2016.

[18] Y.-J. Chang, S.-F. Chen, and J.-D. Huang, "A kinectbased system for physical rehabilitation: A pilot study for young adults with motor disabilities," $R e$ search in developmental disabilities, vol. 32, no. 6, pp. 2566-2570, 2011.

[19] C.-Y. Chang, B. Lange, M. Zhang, S. Koenig, P. Requejo, N. Somboon, A. A. Sawchuk, A. A. Rizzo, et al., "Towards pervasive physical rehabilitation using microsoft kinect.," in PervasiveHealth, pp. 159$162,2012$.

[20] D. Sadihov, B. Migge, R. Gassert, and Y. Kim, "Prototype of a vr upper-limb rehabilitation system enhanced with motion-based tactile feedback," in World Haptics Conference (WHC), 2013, pp. 449454, IEEE, 2013.

[21] X. H. Bao, Y. Mao, Q. Lin, Y. Qiu, S. Chen, L. Li, R. S. Cates, S.-F. Zhou, and D. Huang, "Mechanism of kinect-based virtual reality training for motor functional recovery of upper limbs after subacute stroke," in Neural regeneration research, 2013.

[22] J. M. I. Zannatha, A. J. M. Tamayo, A. D. G. Sánchez, J. E. L. Delgado, L. E. R. Cheu, and W. A. S. Arévalo, "Development of a system based on $3 \mathrm{~d}$ vision, interactive virtual environments, ergonometric signals and a humanoid for stroke rehabilitation," Computer Methods and Programs in Biomedicine, vol. 112, no. 2, pp. 239 - 249, 2013. SI: Computer Assisted Tools for Medical Robotics.

[23] J.-A. Lozano-Quilis, H. Gil-Gomez, J.-A. GilGómez, S. Albiol-Perez, G. Palacios, H. M. Fardoum, and A. S. Mashat, "Virtual reality system for multiple sclerosis rehabilitation using kinect," in Proceedings of the 7th International Conference on Pervasive Computing Technologies for Healthcare, pp. 366-369, ICST (Institute for Computer Sciences, Social-Informatics and Telecommunications Engineering), 2013.

[24] M. Trombetta, P. P. B. Henrique, M. R. Brum, E. L. Colussi, A. C. B. De Marchi, and R. Rieder, "Motion rehab ave 3d: a vr-based exergame for post-stroke rehabilitation," Computer methods and programs in biomedicine, vol. 151, pp. 15-20, 2017.

[25] S. Simmons, R. McCrindle, M. Sperrin, and A. Smith, "Prescription software for recovery and rehabilitation using microsoft kinect," in Pervasive Computing Technologies for Healthcare (PervasiveHealth), 2013 7th International Conference on, pp. 323-326, IEEE, 2013.

[26] R. A. Clark, Y.-H. Pua, A. L. Bryant, and M. A. Hunt, "Validity of the microsoft kinect for providing lateral trunk lean feedback during gait retraining," Gait $\mathcal{F}$ Posture, vol. 38, no. 4, pp. 1064 - 1066 , 2013. 
[27] S. Spasojević, N. V. Ilić, A. Rodić, and J. SantosVictor, "Kinect-based application for progress monitoring of the stroke patients," in Proceedings of IcETRAN conference, vol. ROI2, vol. 6, pp. 1-5, 2017.

[28] M. Antunes, R. Baptista, G. Demisse, D. Aouada, and B. Ottersten, "Visual and human-interpretable feedback for assisting physical activity," in European Conference on Computer Vision (ECCV) Workshop on Assistive Computer Vision and Robotics Amsterdam, 2016.

[29] R. Baptista, M. Antunes, A. E. R. Shabayek, D. Aouada, and B. Ottersten, "Flexible feedback system for posture monitoring and correction," in IEEE International Conference on Image Information Processing (ICIIP), 2017.

[30] R. Baptista, E. Ghorbel, A. E. R. Shabayek, D. Aouada, and B. Ottersten, "Key-skeleton based feedback tool for assisting physical activity," in 2018 Zooming Innovation in Consumer Technologies Conference (ZINC), pp. 175-176, IEEE, 2018.

[31] M. Antunes, D. Aouada, and G. Demisse, "Physical activity feedback," Patent WO2017207802A1, 2017.

[32] A. E. R. Shabayek, R. Baptista, K. Papadopoulos, G. Demisse, O. Oyedotun, M. Antunes, D. Aouada, B. Ottersten, M. Anastassova, M. Boukallel, S. Panels, G. Randall, M. Andre, A. Douchet, S. Bouilland, and L. O. Fernandez, "Starr - decision support and self-management system for stroke survivors vision based rehabilitation system," in European Project Space on Networks, Systems and Technologies - Volume 1: EPS Porto 2017,, pp. 69-80, INSTICC, SciTePress, 2017.

[33] E. Ghorbel, R. Boutteau, J. Boonaert, X. Savatier, and S. Lecoeuche, "Kinematic spline curves: A temporal invariant descriptor for fast action recognition," Image and Vision Computing, 2018.

[34] G. Kwakkel, B. J. Kollen, and H. I. Krebs, "Effects of robot-assisted therapy on upper limb recovery after stroke: a systematic review," Neurorehabilitation and neural repair, 2007.
[35] F. Ofli, G. Kurillo, S. Obdrzálek, R. Bajcsy, H. B. Jimison, and M. Pavel, "Design and evaluation of an interactive exercise coaching system for older adults: Lessons learned," IEEE J. Biomedical and Health Informatics, 2016.

[36] J. M. Veerbeek, E. van Wegen, R. van Peppen, P. J. van der Wees, E. Hendriks, M. Rietberg, and G. Kwakkel, "What is the evidence for physical therapy poststroke? a systematic review and metaanalysis," PloS one, 2014.

[37] P. Andlin-Sobocki, B. Jönsson, H.-U. Wittchen, and J. Olesen, "Cost of disorders of the brain in europe," European Journal of Neurology, 2005.

[38] P. Langhorne, G. Taylor, G. Murray, M. Dennis, C. Anderson, E. Bautz-Holter, P. Dey, B. Indredavik, N. Mayo, M. Power, et al., "Early supported discharge services for stroke patients: a meta-analysis of individual patients' data," The Lancet, 2005.

[39] D. D. N. Natta, E. Alagnide, G. T. Kpadonou, G. G. Stoquart, C. Detrembleur, and T. M. Lejeune, "Feasibility of a self-rehabilitation program for the upper limb for stroke patients in benin," Annals of Physical and Rehabilitation Medicine, vol. 58, no. 6, pp. 322 $-325,2015$.

[40] C. Bonnyaud, P. Gallien, P. Decavel, P. Marque, C. Aymard, F. Pellas, M.-E. Isner, F. C. Boyer, F. Muller, J.-C. Daviet, P. Dehail, B. Perrouin-Verbe, N. Bayle, E. Coudeyre, D. Perennou, I. Laffont, J. Ropers, N.-Y. Domingo-Saidji, D. Bensmail, and N. Roche, "Effects of a 6-month self-rehabilitation programme in addition to botulinum toxin injections and conventional physiotherapy on limitations of patients with spastic hemiparesis following stroke (adju-tox): protocol study for a randomised controlled, investigator blinded study," BMJ Open, vol. 8 , no. $8,2018$.

[41] F. Spyridonis, J. Gawronski, G. Ghinea, and A. O. Frank, "An interactive 3-d application for pain management: Results from a pilot study in spinal cord injury rehabilitation," Computer methods and programs in biomedicine, vol. 108, no. 1, pp. 356-366, 2012. 
[42] H. Pirsiavash, C. Vondrick, and A. Torralba, "Assessing the quality of actions," in Computer VisionECCV 2014, pp. 556-571, Springer, 2014.

[43] M. F. Levin, J. A. Kleim, and S. L. Wolf, "What do motor recovery and compensation mean in patients following stroke?," Neurorehabilitation and Neural Repair, vol. 23, no. 4, pp. 313-319, 2009. PMID: 19118128.

[44] M. F. Levin, S. M. Michaelsen, C. M. Cirstea, and A. Roby-Brami, "Use of the trunk for reaching targets placed within and beyond the reach in adult hemiparesis," Experimental Brain Research, vol. 143, pp. 171-180, Mar 2002.

[45] A. Roby-Brami, S. Jacobs, N. Bennis, and M. F. Levin, "Hand orientation for grasping and arm joint rotation patterns in healthy subjects and hemiparetic stroke patients," Brain Research, vol. 969, no. 1, pp. $217-229,2003$.

[46] R. Wang, G. Medioni, C. Winstein, and C. Blanco, "Home monitoring musculo-skeletal disorders with a single 3d sensor," in Proceedings of the IEEE Conference on Computer Vision and Pattern Recognition Workshops (CVPRW), 2013.

[47] R. Vemulapalli, F. Arrate, and R. Chellappa, "Human action recognition by representing $3 \mathrm{~d}$ skeletons as points in a lie group," in Proceedings of the IEEE Conference on Computer Vision and Pattern Recognition (CVPR), 2014.

[48] J. L. McGinley, R. Baker, R. Wolfe, and M. E. Morris, "The reliability of three-dimensional kinematic gait measurements: a systematic review," Gait $\mathcal{E}$ posture, vol. 29, no. 3, pp. 360-369, 2009.

[49] A. E. F. D. Gama, T. M. Chaves, L. S. Figueiredo, A. Baltar, M. Meng, N. Navab, V. Teichrieb, and P. Fallavollita, "Mirrarbilitation: A clinically-related gesture recognition interactive tool for an ar rehabilitation system," Computer Methods and Programs in Biomedicine, vol. 135, pp. 105 - 114, 2016.

[50] L. Tao, A. Paiement, D. Aldamen, M. Mirmehdi, S. Hannuna, M. Camplani, T. Burghardt, and
I. Craddock, "A comparative study of pose representation and dynamics modelling for online motion quality assessment," Computer Vision and Image Understanding, vol. 11, 2016.

[51] R. Baptista, G. Demisse, D. Aouada, and B. Ottersten, "Deformation-based abnormal motion detection using 3d skeletons," in 2018 Eighth International Conference on Image Processing Theory, Tools and Applications (IPTA), pp. 1-6, IEEE, 2018. 